\title{
Self-assembly of Janus particles confined in a channel
}

\author{
M. Sobrino Fernández, ${ }^{*}$ V. R. Misko, ${ }^{\dagger}$ and F. M. Peeters ${ }^{\ddagger}$ \\ Departement Fysica, Universiteit Antwerpen, B-2020 Antwerpen, Belgium
}

(Received 8 October 2013; published 21 February 2014)

\begin{abstract}
Janus particles present an important class of building blocks for directional assembly. These are compartmentalized colloids with two different hemispheres. Here, we consider a two-dimensional model of Janus disks consisting of a hydrophobic semicircle and an electro-negatively charged one. Placed in a solution, the hydrophobic sides will attract each other while the charged sides will give rise to a repulsive force. Using molecular dynamics simulations, we study the morphology of these particles when confined in a channel-like environment using a one dimensional harmonic confinement potential. The interest to this system is first of all due to the fact that it could serve as a simple model for membrane formation. Indeed, the recently synthesized new class of artificial amphiphiles, known as Janus dendrimers, were shown to self-assemble in bilayer structures mimicking biological membranes. In turn, Janus particles that combine the amphiphilicity and colloidal rigidity serve as a good model for Janus dendrimers. A variety of ordered membrane-like morphologies are found consisting of single and multiple chain configurations with different orientations of the particles with respect to each other that we summarize in a phase diagram.
\end{abstract}

DOI: 10.1103/PhysRevE.89.022306

PACS number(s): 82.70.Dd, 64.75.Yz, 87.16.D-

\section{INTRODUCTION}

Fabrication of ordered arrays of nanoparticles is of significance for fundamental science associated with both lowdimensional physics and its applications. Up-to-date lithography is the major technique in generating arrays. This technique is fairly expensive, complicated, and time consuming. As an alternative route to this top-down approach is a bottom-up selfassembly. Monodisperse spheres of submicron size can readily self-assemble into highly ordered and close-packed arrays, so-called colloidal crystals [1]. Most of these early studies were restricted to isotropic interactions and therefore the possible structures that can be realized were limited. Recently, patchy colloidal particles have attracted a large amount of interest. These are colloids that have an anisotropic interparticle interaction. Different experimental techniques are available to locally modify the chemical composition and the shape of colloidal particles [2-5]. These local modifications are called patches, and depending on the number, size, and chemical properties of each patch these colloids exhibit extraordinary diffusive properties [6,7] or can be used as building blocks for complex structures [8-10]. The substantial research effort in preparing these nanoparticles is driven by their potential applications in nanoelectronics [11], drug delivery [12,13], liquid optics [14,15], separation of mixtures [16], etc. Janus particles are colloids that consist of two hemispheres that have different chemical properties. The preparation of Janus nanoparticles is still a major challenge, but different methods have been reported in the literature $[17,18]$.

In our work, we model Janus spheres by two-dimensional disks that consist of two semicircles: a hydrophobic side, and an electro-negatively charged one. The fabrication of these colloidal spheres was achieved several years ago by depositing a monolayer of octadecanethiol (ODT) on polystyrene spheres

\footnotetext{
*mario.sobrinofernandez@uantwerpen.be

†vyacheslav.misko@uantwerpen.be

${ }^{\ddagger}$ francois.peeters@uantwerpen.be
}

with a thin film of gold on one hemisphere [19]. When placed in a solution, the hydrophobic sides will attract each other at short distances between the two semicircles, while the electrostatic repulsion will repel the particles. In the experiment, depending on the ion concentration (ionic strength) of the solution, different assembled structures can be expected. For low ionic strength, the electrostatic interaction will make the colloids repel each other regardless of their relative orientation. By increasing the ionic strength of the solution, the screening length of the repulsion can be controlled so that the repulsion between two particles will only be of significance when two charged semicircles touch one another (see Fig. 1). The phase diagram and dynamic properties of Janus particles have been studied previously in great detail $[20,21]$. How their properties are modified when such particles are confined in a channel-like environment is not yet known. The interest to this system is first of all due to the fact that it could serve as a simple model for membrane formation. Indeed, the recently synthesized new class of artificial amphiphiles, known as Janus dendrimers [22], were shown to self-assemble in bilayer structures mimicking biological membranes. In turn, Janus particles that combine the amphiphilicity and colloidal rigidity serve as a good model for Janus dendrimers. Therefore, we analyze here the morphologies of Janus disks in a two-dimensional (2D) plane, which are trapped in a one-dimensional harmonic potential. (It is worth noting that although real three-dimensional Janus particles possess 3D orientational degrees of freedom, the $2 \mathrm{D}$ model of disks catches the main features of the specific $2 \mathrm{D}$ patterns since these are in-plane orientations which mainly contribute to structures formed in two dimensions.) We investigate the interplay between the strength of the interparticle interaction, the strength of the 1D confinement and the density of the particles. Using this system we study the dynamics of Janus particles in a quasi-one-dimensional environment by means of molecular dynamics simulations. Chain-like morphologies are of particular interest in the development of electronic appliances, such as a single-electron transistor [23] and high-efficiency photoanodes [24]. The paper is organized as 

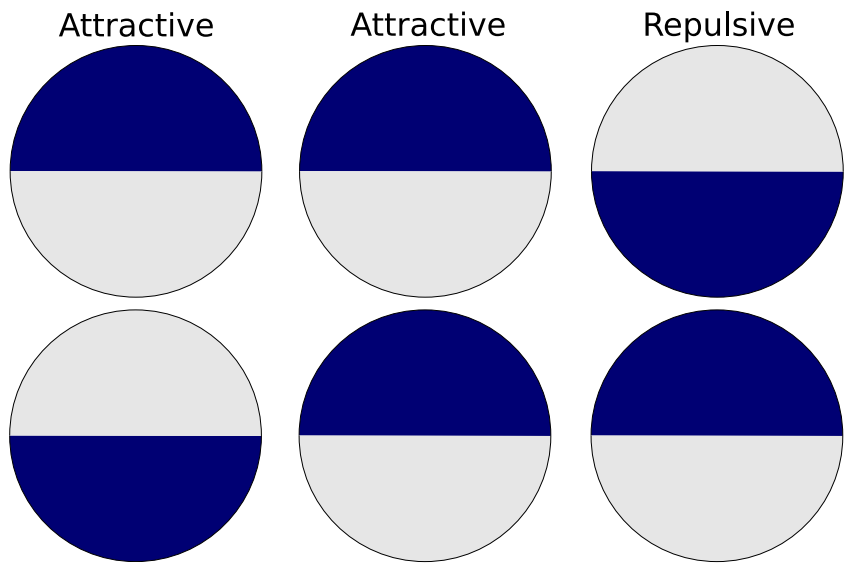

FIG. 1. (Color online) Different configurations of adjoining Janus particles in a solution. The anisotropic potential $\phi^{I}$ [see Eq. (3)] has an attractive contribution if a hydrophobic patch is involved in the interaction. If charged patches face each other, the colloids will repel each other.

follows. In Sec. II we discuss the underlying model for Janus particle dynamics, the simulation method, and the parameters used. Section III is devoted to the numerical results of the molecular dynamics simulations. We start by determining equilibrium configurations of the system, followed by the proposal of a classification scheme that is used to construct a phase diagram. Finally the conclusions are given in Sec. IV.

$$
\begin{aligned}
& \phi^{I}\left(\boldsymbol{r}_{\mathbf{1 2}}, \hat{\boldsymbol{u}_{1}}, \hat{\boldsymbol{u}_{\mathbf{2}}}\right)=-\frac{\kappa_{H}}{2}\left(2 \sigma_{H}-r_{12}\right)^{2}, \\
& \phi^{I}\left(\boldsymbol{r}_{\mathbf{1 2}}, \hat{\boldsymbol{u}_{\mathbf{1}}}, \hat{\boldsymbol{u}_{\mathbf{2}}}\right)=Z^{2} \lambda_{B}\left(\frac{\exp (\kappa \sigma)}{1+\kappa \sigma}\right)^{2} \frac{\exp (-\kappa r)}{r},
\end{aligned}
$$

$\kappa_{H}$ and $\sigma_{H}$ are elastic parameters similar to the ones used in Eq. (2); they measure the strength and range of the attraction. The electrostatic repulsion of colloids in a solution is governed by a screened Coulomb potential given by the DerjaguinLandau-Verwey-Overbeek (DLVO) [28] theory. $Z$ is the effective charge number of the colloidal particles, $\lambda_{B}$ is the Bjerrum length, $\kappa^{-1}$ is the Debye-Hückel screening length which depends on the solvent, and $\beta^{-1}=k_{B} T$ is the thermal energy of the system at temperature $T$ with $k_{B}$ the Boltzmann constant.

We perform 2D molecular dynamics simulations using a computational unit cell of 200 particles confined in a one-dimensional parabolic potential $V(y)=\frac{1}{2} K y^{2}$ and apply periodic boundary conditions in the perpendicular direction, forcing the colloids in a quasi-one-dimensional state. We study the motion of the Janus particles by using a Verlet algorithm. The temperature of the solution remains fixed. The applied temperature is solely used to control the diffusivity coefficient of the colloids $D=\mu k_{B} T$. Parameters are chosen such that particles hardly interpenetrate, and so that the electrostatic repulsion can be neglected when two charged patches do not touch each other (see Fig. 1). If the solvent has a weak ionic strength, the electrostatic repulsion is so strong that particles repel each other regardless of their relative orientation. This effect weakens strongly as soon as the Debye-Hückel screening length $\kappa^{-1}$ becomes smaller than the particle size. At this

\section{MODEL AND SIMULATION TECHNIQUE}

We focus on a system of soft disks in a two-dimensional plane. Each disk consists of two semicircles, one being hydrophobic and the other being negatively charged. The total pair potential between the two disks placed at positions $\boldsymbol{r}_{\mathbf{1}}$ and $\boldsymbol{r}_{\mathbf{2}}$ can be written down in two distinct parts, an isotropic term representing the soft-disk repulsion and an angle-dependent factor characterizing the patchy structure of the colloids,

$$
\phi\left(\boldsymbol{r}_{\mathbf{1 2}}, \hat{\boldsymbol{u}_{\mathbf{1}}}, \hat{\boldsymbol{u}_{2}}\right)=\phi^{S}\left(r_{12}\right)+\phi^{I}\left(\boldsymbol{r}_{\mathbf{1 2}}, \hat{\boldsymbol{u}_{\mathbf{1}}}, \hat{\boldsymbol{u}_{\mathbf{2}}}\right)
$$

where $\boldsymbol{r}_{\mathbf{1 2}}=\boldsymbol{r}_{\mathbf{1}}-\boldsymbol{r}_{\mathbf{2}}$ and $\hat{\boldsymbol{u}}_{\boldsymbol{i}}$ are the polar axes indicating the centers of the hydrophobic patches. As a simple model for soft-disk repulsion, we use an elastic repulsion between two overlapping disks [25]:

$$
\begin{array}{ll}
\phi^{S}\left(r_{12}\right)=\frac{\epsilon}{2}\left(2 \sigma-r_{12}\right)^{2}, & r_{12}<2 \sigma \\
\phi^{S}\left(r_{12}\right)=0, & r_{12}>2 \sigma
\end{array}
$$

where $\epsilon$ characterizes the flexibility of the disks, and $\sigma$ is the disk's radius. An elastic repulsion $[21,25,26]$ is preferred over the hard wall potential proposed by the Kern-Frenkel model [27]. This will facilitate the usage of molecular dynamics simulations, while still having the possibility of tuning the potential so that particles hardly overlap. The anisotropic contribution $\phi^{I}$ depends on the relative orientation between two particles (see Fig. 2):

$$
\begin{aligned}
& r_{12}<2 \sigma_{H} \wedge \hat{\boldsymbol{u}}_{1} \cdot \hat{\boldsymbol{r}}_{12} \geqslant 0 \| \hat{\boldsymbol{u}}_{2} \cdot \hat{\boldsymbol{r}}_{21} \geqslant 0, \\
& \hat{\boldsymbol{u}}_{1} \cdot \hat{\boldsymbol{r}}_{12} \leqslant 0 \wedge \hat{\boldsymbol{u}}_{2} \cdot \hat{\boldsymbol{r}}_{21} \leqslant 0 .
\end{aligned}
$$

point more particle orientations become energetically stable and more structures can be obtained. Parameters are chosen similar to those used in Ref. [19], unless stated otherwise: $\sigma=1 \mu \mathrm{m}, D=0.2 T^{*} \mu \mathrm{m}^{2} / \mathrm{s}, \epsilon=0.8 \mathrm{~N} / \mu \mathrm{m}, \sigma_{H}=1.15 \mu \mathrm{m}$, $\kappa_{H}=0.1 \mathrm{~N} / \mu \mathrm{m}, Z=0.32 e, \lambda_{B}=2 \mu \mathrm{m}, \kappa=1.4 \mu \mathrm{m}^{-1}$, and $\Delta t=0.05 W \mathrm{~s}$.

\section{NUMERICAL RESULTS}

As a starting point in the determination of the equilibrium configurations and resulting phase diagram we aim to

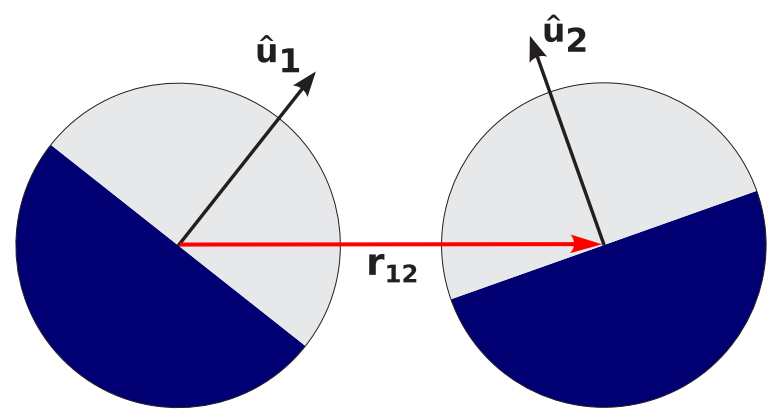

FIG. 2. (Color online) Configuration of two Janus disks. The polar axes $\hat{\boldsymbol{u}}_{1}$ and $\hat{\boldsymbol{u}}_{2}$ indicate the centers of the hydrophobic patches. 
determine the minimum harmonic trap constant $K$ for which aggregation occurs on a large scale, since we are not interested in micelle formation inside the quasi-one-dimensional channel. Our aim is to focus on the aggregation behavior of the system depending on the packing fraction $\eta=N_{\text {part }} \pi \sigma^{2} / L^{2}$, where $N_{\text {part }}$ is the number of particles in a square simulation box with length $L$.

For molecular fluids it is convenient to introduce several types of distribution functions, correlation functions, and related quantities in order to quantify different states. Once, for example, the total correlation function $g\left(\boldsymbol{r}^{h}, \hat{\boldsymbol{u}}^{h}\right)$ for a given system is known, thermodynamic properties of the system can be obtained [29]. To study the equilibrium properties of a general potential, the pair correlation function $g(12)=g\left(\boldsymbol{r}_{1} \boldsymbol{r}_{2} \hat{\boldsymbol{u}}_{1} \hat{\boldsymbol{u}}_{2}\right)$ gives complete information about pairs of molecules [29]. We consider the spherical harmonic coefficients $g_{l_{1} l_{2} l}\left(r_{12}\right)$ of $g(12)$ to highlight particular aspects of the function $g(12)$. The different morphologies are characterized by their density $g_{000}$ and angular pair correlation functions $g_{110}$ :

$$
\begin{gathered}
g_{000}(r)=\frac{L}{2 r N} \sum_{i} \sum_{j \neq i} \delta\left(r-r_{i j}\right), \\
g_{110}(r)=\frac{L}{2 r N} \sum_{i} \sum_{j \neq i} \delta\left(r-r_{i j}\right) \hat{\boldsymbol{u}}_{1} \cdot \hat{\boldsymbol{u}}_{2} .
\end{gathered}
$$

The density correlation function (4) is used to characterize the density profile of the structure, the angular pair correlation function (5) represents the relative orientation of two particles inside the cluster. Positive values of $g_{110}\left(r, \hat{\boldsymbol{u}}_{1}, \hat{\boldsymbol{u}}_{2}\right)$ represent an average parallel orientation between two particles, while a negative value expresses an antiparallel orientation. Depending on the packing fraction and external potential, a wide variety of morphologies can be found (see Fig. 3). The largest one-dimensional periodic structure in the trap is an aligned chain where all particles are orientated in the same direction, effectively nullifying the repulsive patch [Fig. 3(a)]. When increasing $\eta$, it becomes impossible for all the particles to stay in the center of the trap, which results in a zig-zag transition to a membrane structure [Fig. 3(b)], driven by the minimization of the transverse trap potential. When further increasing the packing fraction we find a succession of close-packed oriented chains and zig-zag variants. Each of the found stable structures succeeds in canceling out the Coulomb repulsion at small particle distances.

Each structure can be characterized by the pair correlation functions, Eqs. (4) and (5), discussed previously. In Fig. 4 the characteristic correlation functions are shown for different temperatures for three values of packing fraction $\eta: \eta=0.019$ (zig-zag 1-chain or membrane), $\eta=0.042$ (zig-zag 2-chain), and $\eta=0.070$ (oriented 3-chain) which correspond to cases (b), (d), and (e) in Fig. 3. When increasing temperature and thus the mobility of particles in the cluster, the complex gradually loses its positional and orientational order, until the thermal forces become larger than the Janus-particle interactions, which dismantles the structure. The oscillating character of the correlation functions is due to (i) the periodicity of the structure and (ii) orientational interactions. The zig-zag 2-chain and oriented 3-chain [cases in Figs. 3(d) and 3(e)] are similar in terms of density correlations. The difference is that (a)

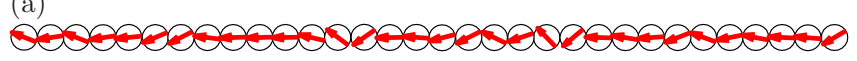

(b)

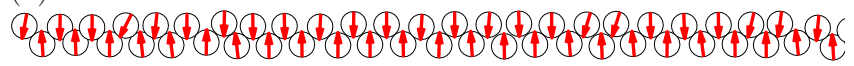

(c)

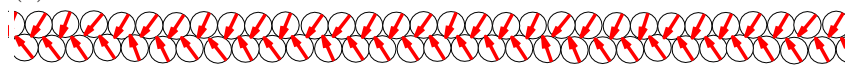

(d)

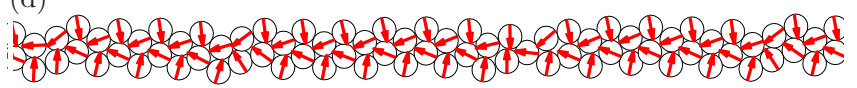

(e)

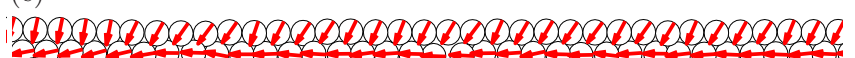
5050000100100000000000001001000

(f)

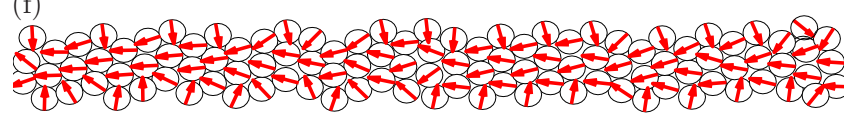

(g)

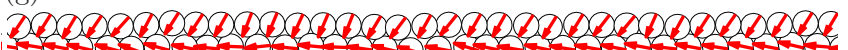

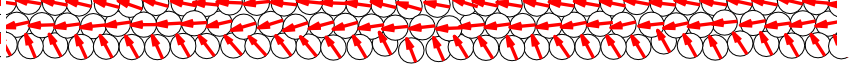

FIG. 3. (Color online) Different particle morphologies of Janus particles in a quasi-1D parabolic confinement potential found with molecular dynamics simulations, depending on the packing fraction $\eta$ and the strength of the transverse trap potential $K \sigma^{2}$ : (a) oriented chain $\left(\eta=0.008, K \sigma^{2}=0.5\right)$, (b) zig-zag 1-chain $(\eta=$ $\left.0.019, K \sigma^{2}=0.5\right)$, (c) oriented 2-chain $\left(\eta=0.031, K \sigma^{2}=0.2\right),(\mathrm{d})$ zig-zag 2-chain $\left(\eta=0.042, K \sigma^{2}=0.2\right)$, (e) oriented 3-chain $(\eta=$ $\left.0.070, K \sigma^{2}=0.2\right)$, (f) zig-zag 3-chain $\left(\eta=0.092, K \sigma^{2}=0.2\right)$, and (g) oriented 4-chain $\left(\eta=0.128, K \sigma^{2}=0.2\right)$. The particle orientation is represented by an arrow pointing toward the hydrophobic patch.

the latter is more ordered since the structure contains more chains [three vs two for Fig. 3(d)]. For the zig-zag 1-chain or membrane [Fig. 3(b)] we find two periodicities for the nearest neighbors: one between particles in the same chain $(r / \sigma=2)$, and one between particles from different chains $(r / \sigma=1)$. Therefore the oscillations in the density correlation function [Fig. 4(a)] have two harmonics. The orientational pair correlation function, on the other hand, loses its oscillating characteristic when increasing the density, as the particles in the cluster consist of stripes with different orientations. The particles in the outer chains of the cluster are oriented like in a membrane, i.e., with the hydrophobic sides inside, while the particles inside the cluster form oriented chains, ensuring that no charged half disks come in close contact with one another [see Figs. 3(d)-3(g)]. Apart from losing its periodic structure, we find that the overall magnitude of $g_{110}$ increases for higher $\eta$. Again, referring to Fig. 3(b), we have a fully antisymmetric, antiferromagnetic (AF)-type, orientation. This property is clearly shown in Fig. 4(b): on average, the orientational correlation is zero since the positive and negative contributions cancel each other. Increasing the density results in the formation of oriented chains inside the cluster [Figs. 3(d)-3(g)], which causes a monotonic rise in the values of $g_{110}$. 

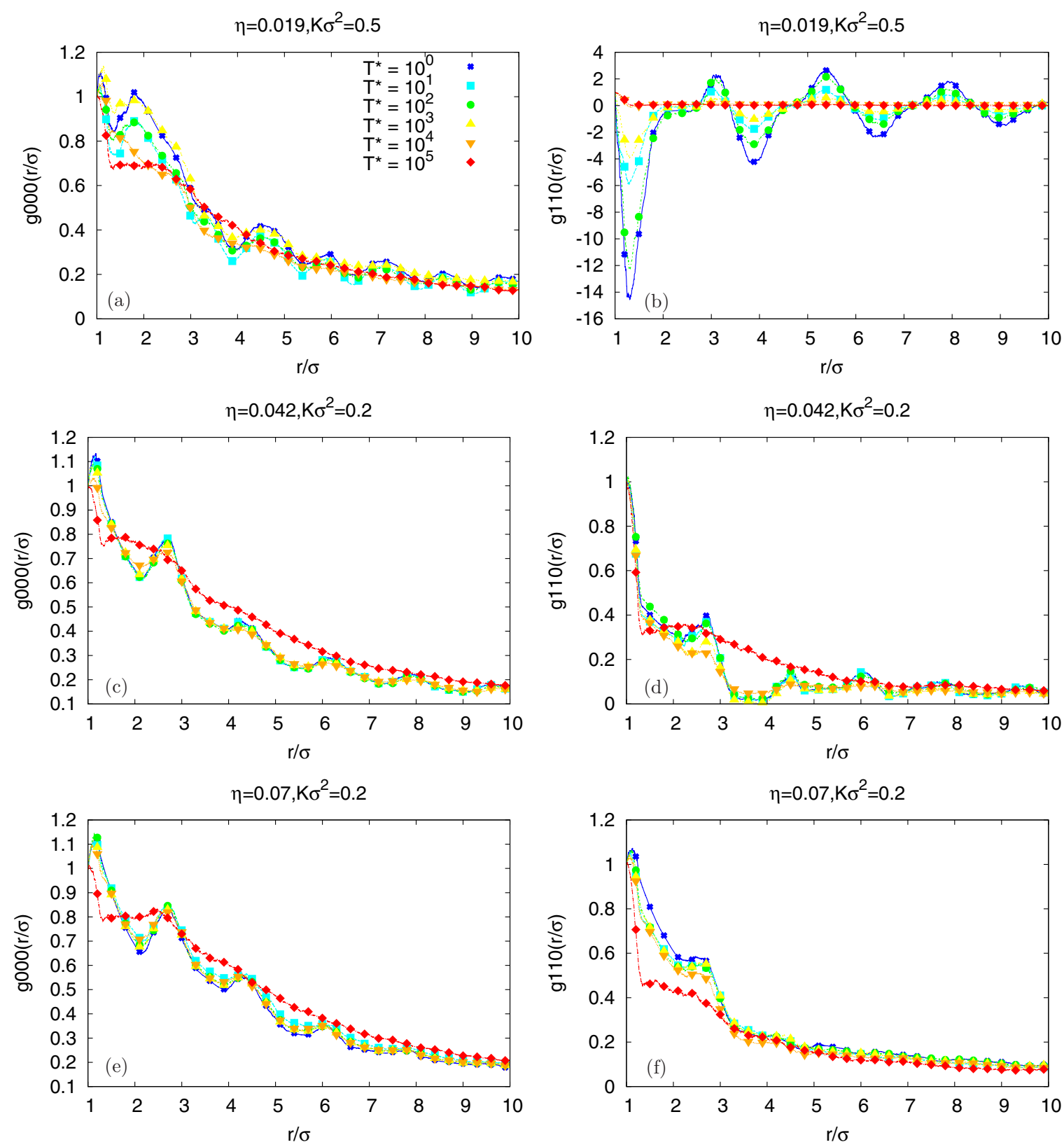

FIG. 4. (Color online) Correlation functions $g_{000}$ and $g_{110}$ for characteristic morphologies at $T^{*}=1,10,10^{2}, 10^{3}, 10^{4}$, and $10^{5}[$ shown by symbols as indicated in (a)] for membranes in (a) and (b), zig-zag 2-chains (c) and (d), and oriented 3-chains (e) and (f). The density profile and angular pair correlation give a quantitative description of each morphology and are used to construct a phase diagram. Each function is normalized at $r / \sigma=1$.

To construct a phase diagram, we need to compose a set of "order parameters" for each structure. A possible approach in achieving this is by defining a set of local pair correlation parameters for each structure:

$$
\begin{aligned}
& \Gamma_{000}=\int_{\sigma}^{3 \sigma} g_{000}(r) d r, \\
& \Gamma_{110}=\int_{\sigma}^{3 \sigma} g_{110}(r) d r,
\end{aligned}
$$

which measures the structural order in the direct vicinity of each particle. For the reference structures the local pair correlation parameters are calculated and plotted in Fig. 5 for $T^{*}=1$. Each intermediate state consists of different areas, where one part of the system is in reference state A, while another part is in state B. For a broad range of values of parameter $\eta$, the functions $\Gamma_{000}$ and $\Gamma_{110}$ both exhibit a gradual increase (i.e., a linear increase followed by saturation) with increasing $\eta$, except for small values of $\eta$ where the function $\Gamma_{110}$ takes values in the vicinity of zero and then shows a sharp drop. This drop in $\Gamma_{110}$ at $\eta \approx 0.01$ corresponds to the transition between the oriented chain [Fig. 3(a)] and zig-zag 1-chain [Fig. 3(b)]. The observed behavior is explained as follows: When increasing the packing fraction, the number of neighboring particles for each colloid increases, and thus 

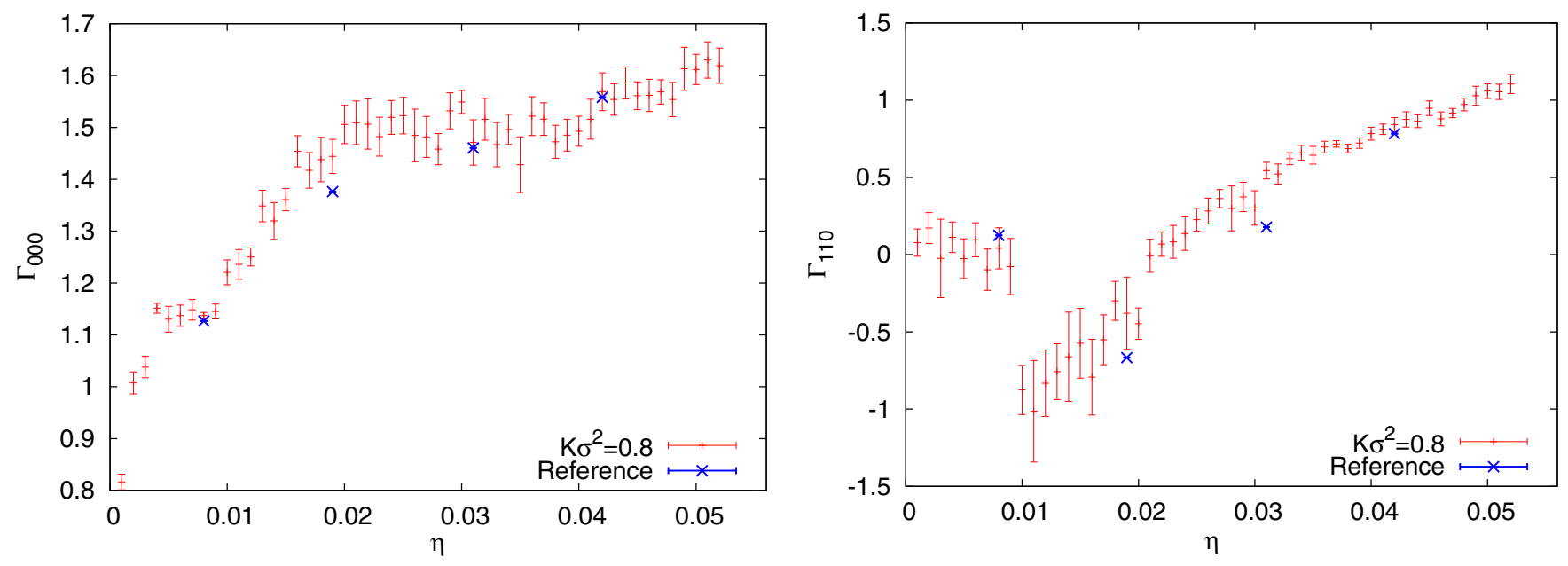

FIG. 5. (Color online) Progression of local pair correlation parameters $\Gamma_{000}$ and $\Gamma_{110}$ for the reference structures displayed in Fig. 3 and intermediate states for a fixed value of the external potential $\left(K \sigma^{2}=0.8\right)$.

the value of $\Gamma_{000}$ increases as well. The linear increase in $\Gamma_{000}$ (for $\eta=0$ to 0.02 ) corresponds to 1-chain and zig-zag 1-chain configurations, when adding new particles results in the linear growth of the number of neighbors for each particle. The value $\eta \approx 0.02$ corresponds to the formation of a 2 -chain configuration, i.e., the appearance of the hexagonal order. For further increasing $\eta$, new particles form additional layers but the hexagonal order remains thus leading to the saturation in $\Gamma_{000}$. Similarly, the function $\Gamma_{110}$ gradually increases (for $\eta>0.01$ ) since the number of inner (co-oriented) chains grows. The sharp drop in $\Gamma_{110}$ at $\eta \approx 0.01$ is due to the chainto-zig-zag transition when the nearest-neighboring particles are oppositely oriented (AF ordering). Adding new inner chains with increasing $\eta$ gradually destroys the AF ordering of the outer chains leading to the above gradual increase in the function $\Gamma_{110}$. Note that the function $\Gamma_{110}$ also shows linear and saturating trends. The former is the signature of the orientational order of the "all surface" particles (for one or two chains) while the latter is related to the formation of the inner layers where all the particles are co-oriented.

A phase diagram in the $\eta-K$ plane is presented in Fig. 6. The different states and transitions can be visualized by assigning a color to each state depending on the value of $\Gamma_{000}$ and $\Gamma_{110}$. No new phase jumps are present, only gradual transitions between different morphologies [except for the sharp transition between the oriented chain (green) and the zig-zag 1-chain (blue)]. The reason for this is two-fold: local density fluctuations (which are more profoundly visible at low $K$ ) make it possible for the system to be at the same time locally in state A (e.g., zig-zag 1-chain) and B (e.g., oriented 2-chain), and there is a certain degree of freedom in which two morphologies can interchange. We find that the most decisive factor in determining a morphology is the packing fraction $\eta$, whereas each state is stable for a large interval of $K$ values. For $\eta<0.007$ the oriented 1 -chain is the most stable morphology, except for very low confinement strength. Here the hydrophobic attractive strength is larger than the trap potential, resulting in the formation of a membrane. For $\eta>0.007$ it becomes impossible for all the particles to occupy the center of the trap, and different morphologies abruptly arise. For low $K$ an oriented 2-chain can easily be formed, while for higher trapping potential the particles get squeezed into a membrane structure. When increasing the density even farther, the membrane gradually evolves into a closely packed membrane with horizontal orientation, a state which we previously labeled as an oriented 2-chain. Increasing the density even farther limits the diversity in possible structures, resulting in a succession of the most tightly packed structures: 2- and 3-chains and zig-zag variants, with crossover states in between.

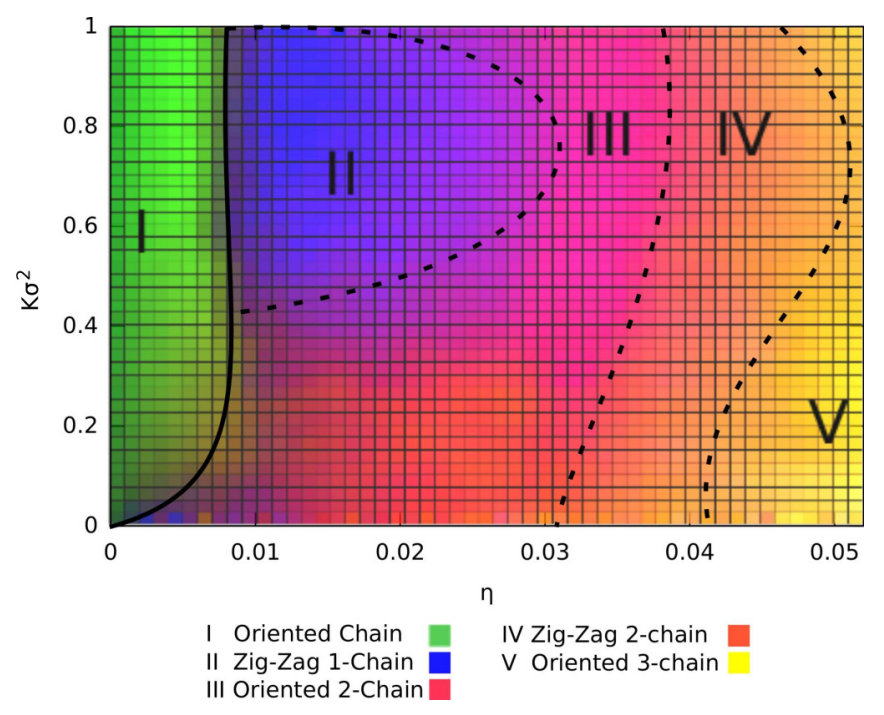

FIG. 6. (Color online) Phase diagram of the different structures formed by Janus disks in a harmonic trap for various values of the packing fraction $\eta$ and confinement strength $K$. Each pure state is represented by a given color: oriented chain (phase I) by green, zig-zag 1-chain (phase II) by blue, oriented 2-chain (phase III) by magenta, zig-zag 2-chain (phase IV) by orange, and oriented 3-chain (phase V) by yellow; mixed (intermediate) states are shown by color gradients. The abrupt transition between the oriented chain and the zig-zag 1-chain and oriented 2-chain is shown by a solid line, while smooth transitions are (approximately) indicated by dashed lines. 


\section{CONCLUSIONS}

We have studied a modified Kern-Frenkel model for soft disks on a two-dimensional plane using molecular dynamics simulations. Each disk consists of a charged semicircle, with one being hydrophobic. The electrostatic repulsion between colloids in solution takes the form of a screened Coulomb potential given by the DLVO theory. For a solvent where the Debye-Hückel screening length is smaller than the particle size, the Janus nature of the colloids becomes profound and a wide range of morphologies are found. These differ in shape and orientational order, which were identified by their density and angular pair correlation footprint. These distinctive features can be used to label each state, and construct a
$\eta-K$ phase diagram. Apart from the abrupt transition between 1D and quasi-1D states, we find smooth crossovers between different morphologies, where increasing the transverse trap potential aids the formation of closely packed and highly ordered states. The Janus particles considered in this work have been already fabricated [19] and we believe that the straightforwardness of the model and its rich phase behavior begs for experimental verifications.

\section{ACKNOWLEDGMENTS}

This work was supported by the Flemish Science Foundation (FWO-Vl) and by the "Odysseus" program of the Flemish government and FWO-Vl.
[1] R. J. Macfarlane, B. Lee, M. R. Jones, N. Harris, G. C. Schatz, and C. A. Mirkin, Science 334, 204 (2011).

[2] A. B. Pawar and I. Kretzschmar, Langmuir 24, 355 (2008).

[3] S. Sacanna, L. Rossi, and D. J. Pine, J. Am. Chem. Soc. 134, 6112 (2012).

[4] S. Sacanna and D. J. Pine, Curr. Opin. Colloid Interface Sci. 16, 96 (2011).

[5] K. P. Yuet, D. K. Hwang, R. Haghgooie, and P. S. Doyle, Langmuir 26, 4281 (2010).

[6] G. Volpe, I. Buttinoni, D. Vogt, H.-J. Kummerer, and C. Bechinger, Soft Matter 7, 8810 (2011).

[7] L. Baraban, R. Streubel, D. Makarov, L. Han, D. Karnaushenko, O. G. Schmidt, and G. Cuniberti, ACS Nano 7, 1360 (2013).

[8] M. J. Solomon, Curr. Opin. Colloid Interface Sci. 16, 158 (2011).

[9] S. C. Glotzer and M. J. Solomon, Nat. Mater. 6, 557 (2007).

[10] G. Doppelbauer, E. Bianchi, and G. Kahl, J. Phys.: Condens. Matter 22, 104105 (2010).

[11] Y. Xiang, S. Lu, and S. P. Jiang, Chem. Soc. Rev. 41, 7291 (2012).

[12] J. Li, C. Fan, H. Pei, J. Shi, and Q. Huang, Adv. Mater. 25, 4386 (2013).

[13] M. J. Campolongo, S. J. Tan, J. Xu, and D. Luo, Adv. Drug Delivery Rev. 62, 606 (2010).

[14] T. Nisisako, T. Torii, T. Takahashi, and Y. Takizawa, Adv. Mater. 18, 1152 (2006).
[15] M. A. Bucaro, P. R. Kolodner, J. A. Taylor, A. Sidorenko, J. Aizenberg, and T. N. Krupenkin, Langmuir 25, 3876 (2009).

[16] W. Yang, V. R. Misko, K. Nelissen, M. Kong, and F. M. Peeters, Soft Matter 8, 5175 (2012).

[17] A. Walther and A. H. E. Muller, Soft Matter 4, 663 (2008).

[18] M. Lattuada and T. A. Hatton, Nano Today 6, 286 (2011).

[19] L. Hong, A. Cacciuto, E. Luijten, and S. Granick, Langmuir 24, 621 (2008).

[20] F. Sciortino, A. Giacometti, and G. Pastore, Phys. Rev. Lett. 103, 237801 (2009).

[21] G. Rosenthal, K. E. Gubbins, and S. H. L. Klapp, J. Chem. Phys. 136, 174901 (2012).

[22] B. M. Rosen, C. J. Wilson, D. A. Wilson, M. Peterca, M. R. Imam, and V. Percec, Chem. Rev. 109, 6275 (2009).

[23] R. S. Gill, R. F. Saraf, and S. Kundu, ACS Appl. Mater. Interfaces 5, 9949 (2013).

[24] S. H. Ko, D. Lee, H. W. Kang, K. H. Nam, J. Y. Yeo, S. J. Hong, C. P. Grigoropoulos, and H. J. Sung, Nano Lett. 11, 666 (2011).

[25] Y. Fily and M. C. Marchetti, Phys. Rev. Lett. 108, 235702 (2012).

[26] P. K. Ghosh, V. R. Misko, F. Marchesoni, and F. Nori, Phys. Rev. Lett. 110, 268301 (2013).

[27] N. Kern and D. Frenkel, J. Chem. Phys. 118, 9882 (2003).

[28] B. V. Derjaguin and L. Landau, Acta Phys. Chim. (USSR) 14, 633 (1941).

[29] C. G. Gray and K. E. Gubbins, Theory of Molecular Fluids, Volume 1: Fundamentals (Oxford University Press, Oxford, 1984). 